Nloman 2021, 39(1)

Revista de Psicologia, Ciències de l'Eduació i de l'Esport

ISSN: $1138-3194$

Facultat de Psicologia, Ciències de l'Educació i de l’Esport Blanquerna

Universitat Ramon Llull

\title{
¿Influyen las respuestas psicológicas y la experiencia en el rendimiento físico del árbitro de fútbol?
}

\author{
Cristóbal Muñoz-Arjona, ${ }^{1}$ Bruno Ricardo Fernandes, ${ }^{2}$ Tito Daniel Dos Santos ${ }^{2}$ \& Alfonso \\ Castillo-Rodríguez ${ }^{1}$ \\ 'Universidad de Granada (España) \\ ${ }^{2}$ Instituto Politécnico de Coimbra (Portugal)
}

Recibido: 2020-9-5

Aceptado: 2021-2-13

doi: 10.51698/aloma.2021.39.1.65-72

¿Influyen las respuestas psicológicas y la experiencia en el rendimiento físico del árbitro de fútbol?

\begin{abstract}
El objetivo de este estudio fue analizar la relación entre variables psicológicas precompetición y respuestas físico-fisiológicas durante la competición de árbitros de fútbol semiprofesionales. Participaron 153 árbitros (edad $\mathrm{M}=23.2 ; \mathrm{SD}=4.8$ años; experiencia $\mathrm{M}=6.0 ; \mathrm{SD}=4.5$ años). Se utilizaron los cuestionarios CSAI- $2 y$ Rosenberg antes del partido y se recogieron datos del rendimiento del partido con dispositivos GPS. Los resultados mostraron que los árbitros experimentados presentaron menores puntuaciones en respuestas psicológicas negativas y mayores, en positivas ( $\mathrm{p}<.01)$. Este grupo desarrolló mayores valores en distancia recorrida y frecuencia cardíaca superior al 95\% de su frecuencia cardíaca máxima $(\mathrm{p}<.05 ; \mathrm{d}>.70)$. Como conclusión, los árbitros experimentados tienen mayor control emocional, que propicia mejores resultados físico-fisiológicos y permite posicionarse más cerca de las jugadas conflictivas, lo que facilita tomar decisiones acertadas. Se recomienda aplicar programas de intervención para ayudar a árbitros más noveles a afrontar la competición e introducir herramientas tecnológicas en categorías nacionales no profesionales, como el videoarbitraje para subsanar errores manifiestos. Todo ello ayudará a los árbitros en su capacidad psico-físico-fisiológica y aumentará su índice de acierto.
\end{abstract}

Palabras clave: experiencia, ansiedad, autoestima, autoconfianza, GPS

Do psychological responses and experience influence the physical performance of soccer referees?

Abstract. The objective of this study was to analyze the relationship between pre-competition psychological vari-
ables and in-match physical-physiological responses of semi-professional soccer referees. One-hundred fifty-three
referees (age $\mathrm{M}=23.2 ; \mathrm{SD}=4.8$ years; experience $\mathrm{M}=6.0 ; \mathrm{SD}=4.5$ years) completed the CSAI-2 and the Rosen-
berg test prior to matches. Physical performance data was collected with GPS devices. The results showed that
experienced referees presented lower scores for the negative psychological responses and higher scores for the positive
ones $(\mathrm{p}<.01)$. During matches, this more experienced group displayed higher values for distance traveled and time
with heart rate greater than $95 \%$ of their maximum heart rate $(\mathrm{p}<.05 ; \mathrm{d}>.70)$. In conclusion, experienced
referees have greater emotional control and better physical-physiological results, which allows them to position
themselves better on the field of play to make correct decisions. The article recommends intervention programs to
help the newer referees to face matches and the introduction of referee-video in non-professional national categories,
to correct clear errors. These changes would help referees to improve in their psycho-physical-physiological capaci-
ties and thus increase their level of success.

Keywords: Experience, anxiety, self-esteem, self-confidence, GPS

Correspondencia

Alfonso Castillo Rodríguez

Departamento de Educación Física y Deportiva

Facultad de Ciencias del Deporte - Universidad de Granada

Crta. Alfacar, s/n. 18011 - Granada (España)

Email: acastillo@ugr.es 


\section{Introducción}

El fútbol es un deporte de naturaleza sumamente compleja, en el que intervienen múltiples factores, por ejemplo, físicos, biomecánicos, fisiológicos, nutricionales, psicológicos,... (Castillo-Rodríguez et al., 2020). Muchos analistas, entrenadores e investigadores dedican un tiempo considerable al estudio del aumento del rendimiento físico (RF), y, a día de hoy, se ofrecen numerosas investigaciones contrastadas (Walker et al., 2019). Por ende, los árbitros de fútbol (AF), evidentemente, son una pieza fundamental en el correcto desempeño de la competición, lo cual ha propiciado el interés de la comunidad científica en las últimas décadas (Mellalieu et al., 2006; Muñoz-Arjona \& Castillo-Rodríguez, 2020). Además, en los últimos años, ha aumentado sustancialmente la exigencia física en competición, lo que requiere un mayor nivel condicional de los jugadores para poder competir en el alto nivel (Bradley et al., 2016) y, por tanto, también de los AF como parte del juego (Medina-Cantalejo, 2017), es decir, el aspecto físico y la composición corporal (Ade et al., 2016), el cuidado alimentario (Jenner et al., 2019; Montesano et al., 2019), el entrenamiento regulado y personalizado (De Campos et al., 2013; Randers et al., 2010) o el control de las variables psicológicas (Jones, 1995; Kais \& Raudsepp, 2005; Montesano et al., 2019; Muñoz-Arjona \& Castillo-Rodríguez 2020), entre otros. Estos condicionantes influyen de manera diferente en la toma de decisiones de los AF durante la competición; el control emocional es uno de los más relevantes (Weinberg \& Richardson, 1990). Diversas investigaciones concluyen incidiendo en la necesidad de implementar programas de intervención para la mejora de las capacidades psicológicas (Loghmani et al., 2018; Muñoz-Arjona \& Castillo-Rodríguez, 2020), las cuales podrían obtener un mayor índice de acierto en el campo, situado actualmente entre el 90 y 95\% (MedinaCantalejo, 2017).

Desarrollar una buena planificación y periodización del entrenamiento podría ayudar a una mejora de la composición corporal (Fernández-García et al., 2019), una reducción de lesiones durante la temporada (Bradley \& Ade, 2018), mejora de estados psicosociales (Onetti-Onetti et al., 2019) y, por ende, se estima que reporte un mayor RF, el cual propicie unos índices de acierto superiores en la toma de decisiones de los partidos, al igual que ocurre con los jugadores de fútbol (Castillo-Rodríguez et al., 2020). El estudio de las variables psicológicas, por ejemplo motivación, atención, estrés, ansiedad, autoconfianza (CO), estados de ánimo, autoestima (AE), miedo a la evaluación social, entre otros (Auweele et al., 1993; Coudevylle et al., 2011; Mesagno et al., 2012), y su incidencia en el RF han sido unos de los principales objetos de estudio de la psicología del deporte (Dosil, 2002), con lo que se concluye que la evaluación y el estudio de dichas variables podrían llegar a garantizar el éxito del deportista (García et al., 2004).

La ansiedad es uno de los constructos más relacionados con el RF (Junge \& Feddermann-Demont, 2016).
Esta se divide en ansiedad cognitiva (AC) y ansiedad somática (AS) (Grossbard et al., 2009; Martens et al., 1990). La primera hace referencia a la dificultad de mantener la concentración; en cambio, la segunda se refiere a las percepciones de los síntomas corporales causados por la activación del sistema nervioso autónomo, como la aceleración del ritmo cardíaco, sudoración, etc. (Martens et al., 1990). Ambos tipos de ansiedad influyen en el RF de manera distinta (Muñoz-Arjona \& Castillo-Rodríguez, 2020). Por un lado, diversos estudios en fútbol no hallaron influencia entre la AC y el RF (Kais \& Raudsepp, 2005; Rodrigo et al., 1990); otros, sin embargo, observaron una relación lineal negativa y una relación de U invertida entre la AS y el RF (RF óptimo en caso de valores de AS medios y menor RF en caso de AS baja y alta) (Craft et al., 2003; Martens et al., 1990; Woodman \& Hardy, 2003); y otros, cuyas investigaciones dieron como resultado una relación directa entre la AC y el RF (Mellalieu et al., 2004) y a la inversa entre la AS y el RF ( $\mathrm{r}=$-.42) (Muñoz-Arjona \& Castillo-Rodríguez, 2020).

Además, la AE es una variable relacionada con la personalidad en la que una persona se valora a sí misma (Molina et al., 2014; Rosenberg, 1965). Asimismo, está relacionada con la salud física (Montero et al., 2012) y psicológica (Sandín et al., 2012), y se asocia positivamente con el bienestar mental de los deportistas por su relación con las características psicológicas positivas (resiliencia, etc.) y emocionales (miedos, ansiedad, depresión, etc.), así como también con la evaluación en situaciones estresantes (Montero et al., 2012; Sandín et al., 2012). Los deportistas con niveles reducidos de $\mathrm{AE}$ tendían a percibir la competición deportiva de una manera más amenazante, mientras que aquellos con unos niveles elevados percibían la competición como una actividad más exigente y obtenían una fuerte relación entre AE y RF (Muñoz-Arjona \& Castillo-Rodríguez, 2020). En el caso de la CO, definida como el grado de certeza que los deportistas poseen en relación con su capacidad para tener éxito en el deporte (Vealey, 1986), numerosas investigaciones han revelado el impacto sobre la práctica deportiva (Ba anac, 2014), pues se ha hallado una relación positiva entre la CO y el RF (Chamberlain y Hale, 2007).

Otro factor que podría modular los estados emocionales de los deportistas es la experiencia, la cual parece estar relacionada con el dominio de diversas variables psicológicas (Rosnet, 2000); los deportistas más experimentados son quienes mayor control emocional poseen (Hanton et al., 2008). En AF semiprofesionales existe una relación negativa entre la experiencia y la AC o la AS (Muñoz-Arjona y Castillo-Rodríguez, 2020), aunque este estudio se centró en las pruebas anuales que tienen los AF para promocionar o descender de categoría. Por otro lado, los AF noveles ofrecen mayores índices de motivación y $\mathrm{CO}$, por lo que podemos afirmar que la experiencia podría influir en el deportista $(r=.64 ; p=.045 ; \mathrm{Ba}$ anac, 2014). Se plantea una hipótesis de partida en la que se estima que los AF experimentados ofrecerán un mayor dominio de las 
respuestas psicológicas (mayores índices de $\mathrm{AE}$ y $\mathrm{CO}$ y menores en ansiedad), las cuales podrían propiciar mayor RF (mayor distancia recorrida, mayor distancia recorrida a velocidades más elevadas, mayor velocidad máxima...) y mayor rendimiento fisiológico (mayor tiempo en umbrales de frecuencia cardíaca por encima del 80-90\%, mayor frecuencia cardíaca máxima, entre otros).

En este sentido, la monitorización, el control y la programación de los parámetros físico-fisiológicos son determinantes con el fin de optimizar el RF y prevenir la aparición de lesiones (Ade et al., 2016; Bradley et al., 2016). Actualmente, la herramienta de monitorización más fiable y utilizada para el control de la carga externa son los sistemas de posicionamiento global (GPS) (Malone et al., 2015; Owen et al., 2017), pues ofrecen una información muy detallada tanto a nivel físico, por ejemplo, distancia total recorrida, distancias y esprints en intervalos de velocidad, cuantificados como frecuencia, porcentaje y minutos, previamente configurados, como a nivel fisiológico, por ejemplo, frecuencia cardíaca (heart rate, HR) mínima (HRMin), media (HRMed), máxima (HRMax) y en diferentes intervalos fijados y calculados a partir de la HRMax. De igual forma, el análisis de la HR viene determinado por el tipo de esfuerzo físico realizado (aceleraciones, desaceleraciones, cambios de dirección, entre otros) que incide en el metabolismo anaeróbico, fundamental en el deporte del fútbol (Woolf et al., 2009).

Por estos motivos, el objetivo de este estudio fue analizar las respuestas psicológicas (AE, AC, AS y CO) previas a los partidos de competición y las respuestas físico-fisiológicas durante los mismos, en AF semiprofesionales en función de la experiencia.

\section{Material y método}

\section{Participantes}

Los participantes del estudio fueron 153 AF adscritos a la Real Federación Andaluza de Fútbol (edad $M=23.2$; $D T=4.8$ años; experiencia $M=6.0 ; D T=4.5$ años). Dicha muestra realizó un entrenamiento regular entre dos y cinco días por semana con una duración aproximada de 60 minutos, en el que se combinaron ejercicios de tecnificación en el terreno de juego, resistencia aeróbica a través de entrenamientos en intervalos y entrenamiento de velocidad y fuerza. Los AF fueron elegidos al azar entre las plantillas arbitrales confeccionadas por el Comité de Árbitros durante las temporadas 2017-2018 y 2018-2019. Los criterios de inclusión del estudio fueron que los AF evaluados estuvieran arbitrando en la Liga de Tercera División del fútbol español, que el partido evaluado fuera completo (90 minutos o más) y sin incidentes (lesiones o situaciones no normales, que la competición tuviera interrupciones largas o no habituales) y que el AF estuviera de acuerdo con los objetivos del estudio y completara todas sus fases (respuestas psicológicas previas y grabación de datos físicos con GPS incorporado).

\section{Instrumentos}

En primer lugar se cumplimentó un cuestionario ad hoc para la recogida de datos sociodemográficos: edad, años de experiencia, lesiones, categoría y años en la categoría. La experiencia se clasificó en dos grupos: el primero, en 10 años o más en el grupo de experimentados y, el segundo, menos de 10 años en el grupo de no experimentados (Muñoz-Arjona y Castillo-Rodríguez, 2020). En segundo lugar, para la obtención de los datos asociados a las variables psicológicas, se utilizaron los siguientes instrumentos:

- El cuestionario Competitive State Anxiety Inventory-2 (CSAI-2) de Martens et al., 1990 se usó para determinar los niveles de AC, AS y CO. Se componía de un total de 27 ítems cuya valoración estaba comprendida entre nada, algo, bastante y mucho, con puntuaciones de uno a cuatro respectivamente. Para la determinación del valor de cada subescala se atendió a la medida de los ítems correspondientes. - El test de Rosenberg para determinar el grado de AE (Atienza et al., 2000; Rosenberg, 1965). Se componía de un total de 10 ítems (cinco positivos y cinco negativos), a los que se les asignó la siguiente calificación: muy de acuerdo (A), de acuerdo (B), en desacuerdo (C) y muy en desacuerdo (D); cada uno de estos tenía una puntuación de uno a cuatro en función de la respuesta empleada dependiendo de si se trataba de un ítem positivo o negativo. Al obtener la puntuación total se categorizó al deportista con baja autoestima si poseía menos de 25 puntos y existían problemas significativos de AE; entre 26 y 29 , si la autoestima era media y no presentaba problemas de AE graves, aunque resultara conveniente mejorarla; y, entre 30 y 40 puntos, si la AE era elevada y esta AE se consideraba adecuada. Por otro lado, los parámetros de carga externa fueron obtenidos a través de dispositivos de sistemas de posicionamiento global (GPS) de cinco hercios (SPIPRO, GPSport, Canberra, Australia). Estos dispositivos fueron incorporados a través de un chaleco ajustado diseñado específicamente; estos no impedían realizar movimientos y fueron validados para la práctica de deportes de interacción como el fútbol (Petersen et al., 2009). Durante los esprints, el rango de fiabilidad varió entre el 2 y el 13\%, con una subestimación de hasta un $4 \%$ en las distancias. Los movimientos de los AF se codificaron en cinco categorías y umbrales de velocidad (Casamichana et al., 2012): caminar (0.1-6.9 $\mathrm{km} \cdot \mathrm{h}^{-1}$ ), correr a baja velocidad $\left(7.0-12.9 \mathrm{~km} \cdot \mathrm{h}^{-1}\right)$, correr a velocidad media (13.0-17.9 $\left.\mathrm{km} \cdot \mathrm{h}^{-1}\right)$, correr a alta velocidad $\left(18.0-20.9 \mathrm{~km} \cdot \mathrm{h}^{-1}\right)$ y esprintar $\left(>21.0 \mathrm{~km} \cdot \mathrm{h}^{-1}\right)$. El número promedio de satélites durante las mediciones fue de $8 \pm 1$. Para el hallazgo de la HR se utilizó el dispositivo Polar S610i (Polar Electro Oy ${ }^{\circledR}$, Helsinki, Finlandia). La HR fue clasificada en distintas zonas, realizando una sensible adaptación de los porcentajes de la HRMax (Fernández-Elías et al., 2017): HR60 (<59.9\%), HR70 (60-69.9\%), HR80 (70-79.9\%), HR90 (80-89.9\%), HR95 (90-94.9\%) y HR96 (> 95\%). La HRMax fue hallada 
mediante la fórmula predeterminada de Tanaka et al. (2001). Para la valoración subjetiva de la carga interna posesfuerzo (RPE) se utilizó la escala CR-10 de Börg (Scherr et al., 2013).

\section{Procedimiento}

Se llevó a cabo un diseño del estudio de carácter transversal (de dos años de duración), no experimental (no se manipularon variables independientes) y de tipo descriptivo e inferencial. En primer lugar, se informó a los AF sobre los objetivos y las acciones que se iban a llevar a cabo en este estudio, y se solicitó consentimiento voluntario firmado antes de iniciar la investigación. Se siguieron las indicaciones establecidas en la Declaración de Helsinki (2013) sobre investigación humana, pues este es un estudio aprobado por el Comité de Ética de la Universidad de Granada (471/ CEIH/2018). Seguidamente, la obtención de las variables psicológicas se realizó diez minutos antes del calentamiento para la competición. Finalizados los mismos, al equipo arbitral se le incorporó dispositivos GPS para la medición del calentamiento y del partido. Una vez el partido hubo concluido, se preguntó sobre la escala de RPE.

\section{Análisis estadístico}

Se utilizó el programa estadístico SPSS para Windows (SPSS Inc., Chicago), versión 25.0. Se realizaron análisis de Kolmogorov-Smirnov para evaluar la normalidad de las variables dependientes. Los resultados mostraron que estas variables seguían una distribución normal. Posteriormente, se llevaron a cabo análisis comparativos ( $t$-test) utilizando la experiencia como variable independiente. El tamaño del efecto ( $d$ de Cohen) se usó para cuantificar el tamaño de la diferencia que se encontró entre ambos grupos. Para el caso de la prueba $t$ de muestras independientes, la $d$ de Cohen se ejecutó como tamaño del efecto utilizando los siguientes cri-

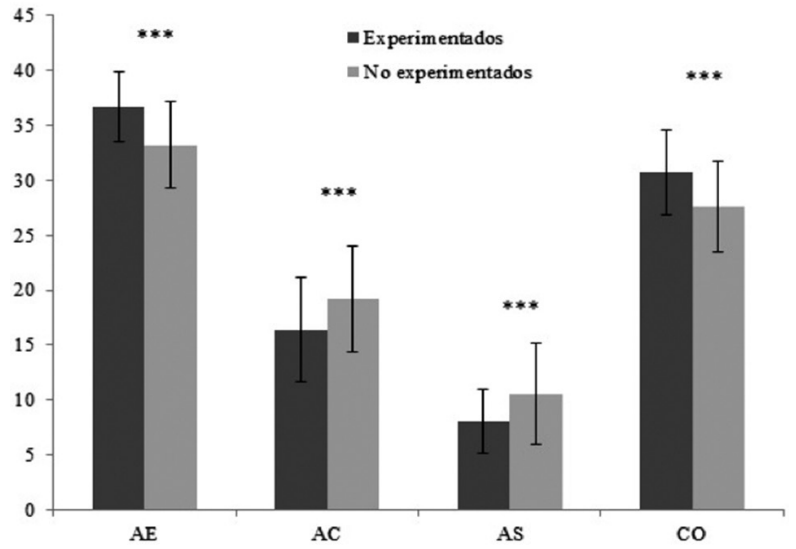

Figura 1. Media \pm desviación típica de las respuestas psicológicas previas a la competición según la experiencia. AE: autoestima; AC: ansiedad cognitiva; AS: ansiedad somática; CO: autoconfianza. A*: autoesti $\mathrm{p}<.001$

terios: efecto pequeño $(d<.20)$, efecto moderado $(.20$ $\leq d<.80)$ y efecto grande $(d \geq .80)$ ( $\mathrm{O}^{\prime}$ Donoghue, 2013). Se definió una significancia estadística del 5\% $(p<.05)$.

\section{Resultados}

En la figura 1 se muestran los resultados de las respuestas psicológicas previas a la competición oficial teniendo en cuenta la experiencia del AF. Se observaron múltiples diferencias significativas (con tamaños del efecto elevados). Los AF experimentados poseían menor ansiedad (AC y AS) y mayor AE y CO $(p<.01 ; d>.60)$.

En la tabla 1 se exponen las respuestas fisiológicas medias atendiendo a la división antes mencionada de la experiencia del AF. Hay que destacar una mayor demanda fisiológica en el grupo experimentado, cuyos valores de las variables HR95 con el 11.3\% y 7.58\% del tiempo de los AF experimentados en la primera y segunda parte respectivamente, frente al $4.80 \%(d=.70)$ y $2.64 \%(d=.75)$ de los AF no experimentados en la primera y segunda parte respectivamente; y en la variable HR96 con el 7.7\% del tiempo de los AF experi-

Tabla 1. Media \pm desviación típica de las respuestas fisiológicas en la competición según la experiencia.

\begin{tabular}{|c|c|c|c|c|c|c|c|c|c|c|}
\hline \multirow[b]{2}{*}{ Partido } & \multirow[b]{2}{*}{$\mathrm{RPE}$} & \multirow[b]{2}{*}{ (puntos) } & \multicolumn{3}{|c|}{$\begin{array}{l}\text { Experimentados } \\
(n=63)\end{array}$} & \multicolumn{3}{|c|}{$\begin{array}{l}\text { No experimentados } \\
(n=90)\end{array}$} & \multirow{2}{*}{$\begin{array}{l}p \\
.115\end{array}$} & \multirow{2}{*}{$\begin{array}{l}d \\
.45\end{array}$} \\
\hline & & & 13.65 & \pm & 2.33 & 12.57 & \pm & 2.41 & & \\
\hline & HRMin & (ppm) & 102.1 & \pm & 16.9 & 102.3 & \pm & 16.1 & .961 & .01 \\
\hline & HRMed & $(\mathrm{ppm})$ & 143.4 & \pm & 22.7 & 141.5 & \pm & 22.3 & .772 & .09 \\
\hline & HRMax & (ppm) & 177.26 & \pm & 23.98 & 175.4 & \pm & 20.9 & .874 & .08 \\
\hline \multirow[t]{6}{*}{ Primera parte } & HR60 & (\%) & 18.61 & \pm & 32.8 & 20.70 & \pm & 33.7 & .839 & .06 \\
\hline & HR70 & $(\%)$ & 18.16 & \pm & 20.0 & 21.91 & \pm & 14.6 & .498 & .22 \\
\hline & HR80 & $(\%)$ & 20.94 & \pm & 16.3 & 29.24 & \pm & 17.5 & .117 & .49 \\
\hline & HR90 & $(\%)$ & 23.54 & \pm & 18.9 & 23.12 & \pm & 17.4 & .978 & .02 \\
\hline & HR95 & (\%) & 11.32 & \pm & 12.5 & 4.795 & \pm & 6.68 & .034 & .70 \\
\hline & HR96 & $(\%)$ & 7.696 & \pm & 12.0 & 0.732 & \pm & 1.25 & .009 & .93 \\
\hline \multirow[t]{6}{*}{ Segunda parte } & HR60 & $(\%)$ & 20.46 & \pm & 30.1 & 23.60 & \pm & 34.0 & .753 & .10 \\
\hline & HR70 & $(\%)$ & 23.63 & \pm & 21.5 & 24.64 & \pm & 15.6 & .865 & .06 \\
\hline & HR80 & $(\%)$ & 23.89 & \pm & 14.4 & 31.15 & \pm & 18.5 & .087 & .43 \\
\hline & HR90 & (\%) & 23.86 & \pm & 20.6 & 17.15 & \pm & 14.8 & .248 & .39 \\
\hline & HR95 & (\%) & 7.577 & \pm & 8.75 & 2.642 & \pm & 4.76 & .029 & .75 \\
\hline & HR96 & $(\%)$ & 2.504 & \pm & 3.96 & 0.847 & \pm & 1.81 & .080 & .58 \\
\hline
\end{tabular}

RPE: carga interna posesfuerzo; HRMin: frecuencia cardíaca mínima; HRMed: frecuencia cardíaca media; HRMax: frecuencia cardíaca máxima; HR60: frecuencia cardíaca inferior a 59.9\%; HR70: frecuencia cardíaca entre 60 y 69.9\%; HR80: frecuencia cardíaca entre 70 y 79.9\%; HR90: frecuencia cardíaca entre 80 y $89.9 \%$; HR95: frecuencia cardíaca entre 90 y 94.9\%; HR96: frecuencia cardíaca superior de $95 \%$. 
Tabla 2. Media \pm desviación típica de las respuestas físicas en la competición según la experiencia

\begin{tabular}{|c|c|c|c|c|c|c|c|c|c|}
\hline \multirow[b]{2}{*}{ DT } & \multirow[b]{2}{*}{ (metros) } & \multicolumn{3}{|c|}{$\begin{array}{l}\text { Experimentados } \\
(\mathrm{n}=63)\end{array}$} & \multicolumn{3}{|c|}{$\begin{array}{l}\text { No experimentados } \\
(\mathrm{n}=90)\end{array}$} & \multirow{2}{*}{$\begin{array}{l}p \\
.103\end{array}$} & \multirow{2}{*}{$\begin{array}{l}d \\
.48 \\
\end{array}$} \\
\hline & & 7770.0 & \pm & 2662.9 & 6589.7 & & 2330.7 & & \\
\hline E_D_345 & (metros) & 1908.2 & \pm & 1428.9 & 1509.1 & \pm & 1175.8 & .292 & .31 \\
\hline E_SP_345 & (número) & 389.1 & \pm & 267.7 & 336.4 & \pm & 283.5 & .507 & .19 \\
\hline ¿_Tiempo_345 & (minutos) & 6.90 & \pm & 4.84 & 5.72 & \pm & 4.47 & .381 & .26 \\
\hline
\end{tabular}

DT: distancia total; $\sum_{\_}$D_345: suma de distancias mayores de $13.0 \mathrm{~km} \cdot \mathrm{h}^{-1} ; \sum_{-}$Tiempo_345: suma de tiempo en velocidad superior $13.0 \mathrm{de} \mathrm{km} \cdot \mathrm{h}^{-1}$

mentados en la primera parte, frente al $0.73 \%(d=.93)$ de los AF no experimentados en la misma parte del partido. Por el contrario, los AF no experimentados se encontraron mayor tiempo en HR80 (primera parte: 29.2\%; segunda parte: $31.2 \%$ ) que los AF experimentados (primera parte: 20.9\%; segunda parte: $23.9 \%$ ), aunque dichas diferencias no fueron significativas $(d=$ .49 y .43 , respectivamente).

Además, en las respuestas físicas en estos AF no se observaron diferencias significativas, aunque se apreciaron unos efectos moderados en la distancia total, y en la primera parte del partido, en las variables de distancias recorridas a partir de $18.0 \mathrm{~km} \cdot \mathrm{h}^{-1}$. Los AF experimentados demandaban mayores distancias en dichas variables $(d>.32)$.

\section{Discusión}

El objetivo del presente estudio fue analizar las respuestas psicológicas (AE, AC, AS, CO) y las respuestas físicas y fisiológicas de los AF semiprofesionales en partidos de competición en función de la experiencia. Los resultados mostraron que los AF tienen respuestas psicológicas diferentes momentos previos de la competición. Esta varianza se muestra entre los AF experimentados y los no experimentados, encontrando un tamaño del efecto muy grande.

Los AF experimentados poseen menor ansiedad y mayor $\mathrm{CO}$ y AE, tanto en valores absolutos como relativos, lo que está en consonancia con los estudios de Hanton et al. (2008), Muñoz-Arjona y Castillo-Rodríguez (2020) y Rosnet (2000), quienes afirman que los deportistas experimentados ofrecen un mayor control emocional. Por lo tanto, los AF se encontrarían en mejores condiciones para la competición (García et al., 2004), podrían llegar a tomar decisiones con más tranquilidad y seguridad (Weinberg \& Richardson, 1990), y evitarían que las respuestas fisiológicas próximas al partido (en los 15 primeros minutos y en los últimos 15 minutos del encuentro), cuando se producen situaciones más decisivas, pudieran elevarse, lo que provocaría fatiga muscular y un descenso del RF (Mallo-Sainz et al., 2006). En este sentido, los AF no experimentados desarrollaron menor distancia total en el partido $(d=$ $.48)$, que podría deberse a una menor AE $(d=.96)$ y CO $(d=.77)$, así como a una mayor AS $(d=.64)$ y AC $(d=$ .60). Por ende, este estudio ratificó la conexión entre las respuestas psicológicas y el RF, tal como afirman Jones y Hanton (1996). Esto explica que los AF experimentados realicen acciones a más alta intensidad, porque su control emocional es mayor y porque poseen índices más bajos de AS y AC, además su $\mathrm{CO}$ y $\mathrm{AE}$ también son más elevadas. Todo ello está en sintonía con lo dispuesto por García et al., 2004, quien sostiene que la evaluación y el estudio de dichas variables podrían llegar a garantizar el éxito del deportista. Además, los AF no experimentados tienen escasos registros de $\mathrm{AE}$, lo que concuerda con el estudio de Guillén y Feltz (2011).

Es destacable que los AF experimentados realizaron más metros y esprints en umbrales superiores a 13.0 $\mathrm{km} \cdot \mathrm{h}^{-1}$ durante más tiempo. Estos hallazgos podrían explicar un mayor RF al tratarse de variables de intensidad media-alta (Mohr et al., 2003) y que la HRMed y HRMax (tanto en el partido como en ambas partes) fueran más elevadas, al igual que en HR95 y HR96, en ambas partes. Estos datos fortalecen el efecto producido en la RPE según la experiencia, lo que concuerda con el estudio de Impellizzeri et al. (2004).

Este estudio presenta diversas limitaciones. En primer lugar, la ausencia de técnicas cinemáticas antes mencionadas y del videoarbitraje (VAR). Estas tecnologías se han utilizado únicamente en el fútbol profesional durante estos últimos años. Esto imposibilita que se pueda llevar a cabo una amplia base de datos de registros de temporadas, ya que se trata de un sistema vanguardista y que podría afectar a las respuestas psicológicas en los AF. A tenor de lo expuesto y como futuras líneas de investigación, se propone la inclusión de metodologías de análisis biomecánico para los AF en la totalidad de competiciones nacionales, con el fin de optimizar su RF y su nivel de acierto. Este último incrementaría con la combinación de técnicas de análisis técnico para el estudio del sistema de juego de equipos y jugadores (Dufour, 1993; Payyappalli \& Zhuang, 2019) y con la implementación de las nuevas tecnologías del videoarbitraje (Errekagorri et al., 2020) en los partidos de las categorías no profesionales del fútbol nacional. La introducción de esta tecnología podría hacer fluctuar las respuestas psicológicas de los $\mathrm{AF}$, en beneficio de la toma de decisiones que se lleva a cabo en la competición oficial, y tendría, además, un efecto disuasorio que provocaría una disminución de faltas y tarjetas, tal y como se analizó en los partidos de la Serie A italiana y de la Bundesliga de Alemania (Lago-Peñas et al., 2019). De igual modo, la eficacia de dicha tecnología queda retractada en la Primera División Española, donde se incrementó el índice de acierto un $4.79 \%$ en penaltis (que se situó en un $98.24 \%$ ) y un $1.11 \%$ en fuera de juego (se obtuvo un $94.64 \%$ ). Dichos datos fueron ofrecidos por el Comité Técnico de Árbitros de la Real Federación Española de Fútbol el 
15 de enero de 2019. Por otro lado, la introducción de metodologías biomecánicas y cinemáticas permitiría realizar un autoanálisis a los AF de su posicionamiento ante la toma de cada una de sus decisiones. Por todo ello, se deduce que ambas herramientas tecnológicas son primordiales para la reducción del nivel de acierto, al igual que lo es el control emocional.

\section{Conclusión}

Los AF experimentados presentan mejores respuestas psicológicas previas a la competición y estas influyen en las demandas físico-fisiológicas del partido, por lo que desarrollan mayores respuestas que los AF no experimentados. Este hecho permitirá posicionarse más cerca de las jugadas conflictivas y, por ende, tomar decisiones en consecuencia. Como aplicación práctica, se recomienda el uso de programas de intervención física y psicológica que ayuden a los árbitros más noveles a afrontar la competición.

\section{Lista de abreviaturas}

AC: ansiedad cognitiva.

AS: ansiedad somática.

AE: autoestima.

AF: árbitro de fútbol.

CO: autoconfianza.

CSAI: cuestionario Competitive State Anxiety Inventory-2.

GPS: sistema de posicionamiento global.

HR: frecuencia cardíaca.

HRMax: frecuencia cardíaca máxima.

HRMed: frecuencia cardíaca media.

HRMin: frecuencia cardíaca mínima.

RF: rendimiento físico.

RPE: carga interna posesfuerzo.

\section{Referencias}

Ade, J., Fitzpatrick, J., \& Bradley, P. S. (2016). Highintensity efforts in elite soccer matches and associated movement patterns, technical skills and tactical actions. Information for position-specific training drills. Journal of Sports Sciences, 34(24), 2205-2214. https://doi.org/10.1080/02640414.2016.1217343

Atienza, F. L., Moreno, Y., \& Balaguer, I. (2000). An Analysis of the Dimensionality of the Rosenberg Self-Esteem Scale in a sample of Valencian Adolescents. Revista de Psicología, 22(12), 29-42.

Auweele, Y. V., De-Cuyper, B., Van-Mele, V., \& Rzewnicky, R. (1993). Elite performance and personality: From description and prediction to diagnosis and Intervention. En R. N. Singer, M. Murphey, y L. K. Tennant (eds.), Handbook of Research on Sport Psychology (pp. 257-299). Macmillan.

Bačanac, L., Milićević-Marinković, B., Kasum, G., \& Marinković, M. (2014). Competitive anxiety, selfconfidence and psychological skills in top athletes with and without disabilities: pilot study. Facta Uni- versitatis, Series: Physical Education and Sport, 59-70. https://doi.org/10.1080/10413209708406488

Bradley, P. S., \& Ade, J. (2018). Are Current Physical Match Performance Metrics in Elite Soccer Fit for Purpose or is the Adoption of an Integrated Approach Needed? International Journal of Sports Physiology and Performance, 1-23. https://doi.org/10.1123/ijspp.2017-0433

Bradley, P. S., Archer, D. T., Hogg, B., Schuth, G., Bush, M., Carling, C., \& Barnes, C. (2016). Tier-specific evolution of match performance characteristics in the English Premier League: it's getting tougher at the top. Journal of Sports Sciences, 34(10), 980-7. https://doi.org/10.1080/02640414.2015.1082614

Casamichana, D., Castellano, J., \& Castagna, C. (2012). Comparing the physical demands of friendly matches and small-sided games in semiprofessional soccer players. Journal of Strength and Conditioning Research, 26, 837-843. https://doi.org/10.1519/ JSC.0b013e31822a61cf

Castillo-Rodríguez, A., Cano-Cáceres, F. J., Figueiredo, A., \& Fernández-García, J. C. (2020). Train Like You Compete? Physical and Physiological Responses on Semi-Professional Soccer Players. International Journal of Environmental Research and Public Health, 17(3), 756. https://doi.org/10.3390/ijerph17030756

Castillo-Rodríguez, A., López-Aguilar, J., \& Alonso-Arbiol, I. (2020). Relación entre respuestas físico-fisiológicas y psicológicas en árbitros de fútbol amateur. Revista de Psicología del Deporte, ahead of print.

Chamberlain, S. T., \& Hale, B. D. (2007). Competitive state anxiety and selfconfidence: Intensity and direction as relative predictors of performance on a golf putting task. Anxiety, Stress \& Coping, 20(2), 197-207. https://doi.org/10.1080/10615800701288572

Coudevylle, G. R., Gernigon, C., \& Martin, K. A. (2011). Self-esteem, self-confidence, anxiety and claimed self-handicapping: A mediational analysis. Psychology of Sport and Exercise, 12, 670-675. https://doi. org/10.1016/j.psychsport.2011.05.008

Craft, L. L., Magyar, T. M., Becker, B. J., \& Feltz, D. L. (2003). The relationship between the competitive state anxiety inventory- 2 and sport performance: A meta-analysis. Journal of Sport and Exercise Psychology, 25(1), 44-65. https://doi.org/10.1123/jsep.25.1.44

De Campos, L. C. C., de Athayde Costa, A., dos Santos, L. T. F., Costa, L. T., Montagner, P. C., Borin, J. P., \& Gorla, J. I. (2013). Effects of training in physical fitness and body composition of the brazilian 5-a-side football team. Revista Andaluza de Medicina del Deporte, 6(3), 91-95. https://doi.org/10.1016/S18887546(13)70041-8

Dosil, J. (2002). The sports psychologist. Advice and intervention. Madrid: Síntesis

Dufour, W. (1993). Computer assisted scouting in soccer. Science and Football II, 160-166.

Errekagorri, I., Castellano, J., Echeazarra, I., \& Lago-Peñas, C. (2020). The effects of the Video Assistant Referee system (VAR) on the playing time, technical-tactical and physical performance in elite soccer. International Journal 
of Performance Analysis in Sport, 20(5), 808-817. do i: $10.1080 / 24748668.2020 .1788350$

Fernández-Elías, V. E., Gómez-López, M. De la Vega, R., \& Clemente-Suárez, V. J. (2017). Physical demands, heart rate response and performance of talent football referees. Medicina dello Sport, 70(4), 447-456. https:// doi.org/10.23736/S0025-7826.17.03076-9

Fernández-García, J. C., Castillo-Rodríguez, A., \& Onetti-Onetti, W. (2019). Influencia del sobrepeso y la obesidad sobre la fuerza en la infancia. Nutrición Hospitalaria, 36(5), 1055-1060. http://dx.doi. org/10.20960/nh.02596

García, A., Estrany, B., \& Cruz, J. (2004). How to collect information in psychology of physical activity and the sport: three examples and some considerations? Cuadernos de Psicología del Deporte, 4(1-2), 129-151.

Grossbard, J. R., Smith, R. E., Smoll, F. L., \& Cumming, S. P. (2009). Competitive anxiety in young athletes: Differentiating somatic anxiety, worry and concentration disruption. Anxiety, Stress and Coping, 22(2), 153-166. https://doi.org/10.1080/10615800802020643 Guillén, F., \& Feltz, D. L. (2011). A conceptual model of referee efficacy. Frontiers in Psychology, 2, 25. https://doi.org/10.3389/fpsyg.2011.00025

Hanton, S., Neil, R., Mellaliu, S. D., \& Fletcher, D. (2008). Competitive experience and performance status: An investigation into multidimensional anxiety and coping. European Journal of Sport Science, 8(3), 143-152. https://doi.org/10.1080/17461390801987984

Impellizzeri, F. M., Rampinini, E., Coutts, A. J., Sassi, A. L. D. O., \& Marcora, S. M. (2004). Use of RPE-based training load in soccer. Medicine \& Science in sports \& exercise, 36(6), 1042-1047. https://doi.org/10.1249/01. MSS.0000128199.23901.2F

Jenner, S. L., Devlin, B. L., Forsyth, A. K., \& Belski, R. (2019). Dietary intakes of professional Australian football league women's (AFLW) athletes during a preseason training week. Journal of Science and Medicine in Sport, 22(11), 1266-1271. https://doi. org/10.1016/j.jsams.2019.06.014

Jones, G. (1995). More than just a game: research developments and issues in competitive anxiety in sport. British Journal of Psychology, 86(4), 449-478. https://doi.org/10.1111/j.2044-8295.1995.tb02565.x

Jones, G., \& Hanton, S. (1996). Interpretation of competitive anxiety symptoms and goal attainment expectancies. Journal of Sport and Exercise Psychology, 18(2), 144-157.

https://doi.org/10.1123/jsep.18.2.144

Junge, A., \& Feddermann-Demont, N. (2016). Prevalence of depression and anxiety in top-level male and female football players. BMJ Open Sport \& Exercise Medicine, 2(1), e000087. https://doi.org/10.1136/ bmjsem-2015-000087

Kais, K., \& Raudsepp, L. (2005). Intensity and direction of competitive state anxiety, self-confidence and athletic performance. Kinesiology, 37(1). 13-20.

Lago-Peñas, C., Rey, E., \& Kalén, A. (2019). How does Video Assistant Referee (VAR) modify the game in elite soccer?. International Journal of Performance
Analysis in Sport, 19(4), 646-653. https://doi.org/10. 1080/24748668.2019.1646521

Loghmani, M., Taylor, T., \& Ramzaninejad, R. (2018). Job characteristics and psychological states of football referees: implications for job enrichment. Managing Sport and Leisure, 1, 1-16. https://doi.org/10.1080/2 3750472.2018.1426488

Mallo-Sainz, J., García-Aranda-Encinar, J. M., \& Navarro-Cabello, E. (2006). Análisis del rendimiento físico de los árbitros de fútbol durante partidos de competición oficial. European Journal of Human Movement, 17, 25-40.

Malone, J. J., Di Michele, R., Morgans, R., Burgess, D., Morton, J. P., \& Drust, B. (2015). Seasonal trainingload quantification in elite English premier league soccer players. International Journal of Sports Physiology and Performance, 10(4), 489-97. https://doi. org/10.1123/ijspp.2014-0352

Martens, R., Vealey, R. S., \& Burton, D. (1990). Competitive Anxiety in Sport. Human Kinetics.

Medina-Cantalejo, L. (2017). Visión del arbitraje profesional en España. En Jiménez-Soto, I. (Eds), Los retos del deporte profesional y profesionalizado en la sociedad actual (pp. 173-192). Editorial Reus.

Mellalieu, S. D., Hanton, S., \& O'Brien, M. (2004). Intensity and direction of competitive anxiety as a function of sport type and experience. Scandinavian Journal of Medicine \& Science in Sports, 14(5), 326-334. https://doi.org/10.1111/j.1600-0838.2004.00389.x

Mellalieu, S. D., Neil, R., \& Hanton, S. (2006). SelfConfidence as a Mediator of the Relationship Between Competitive Anxiety Intensity and Interpretation. Research Quarterly for Exercise \& Sport, 77(2), 263-270. https://doi.org/10.1080/02701367.2006.1 0599359

Mesagno, C., Harvey, J. T., \& Janelle, C. M. (2012). Choking under pressure: The role of fear of negative evaluation. Psychology of Sport and Exercise, 13, 60-68. https://doi.org/10.1016/j.psychsport.2011.07.007

Mohr, M., Krustrup, P., \& Bangsbo, J. (2003). Match performance of high-standard soccer players with special reference to development of fatigue. Journal of Sports Sciences, 21(7), 519-528. https://doi. org/10.1080/0264041031000071182

Molina, J., Chorot, P., Valiente, R. M., \& Sandín, B. (2014). Fear of negative evaluation and self-esteem as predictors of sport performance: The mediational role of anxiety and selfconfidence states. Cuadernos de Psicología del Deporte, 14(3), 57-66. https://doi. org/10.4321/S1578-84232014000300007

Montero, P., Rueda, B., \& Bermúdez, J. (2012). Relationship of type D personality and vital exhaustion with negative emotions and psychological adjustment to heart disease. Revista de Psicopatología y Psicología Clínica, 17(2), 93-106.

Montesano, P., Di Silvestro, M., Cipriani, G., \& Mazzeo, F. (2019). Overtraining syndrome, stress and nutrition in football amateur athletes. Journal of Human Sport and Exercise, 14(4), 957-969. https://doi. org/10.14198/jhse.2019.14.Proc4.58 
Muñoz-Arjona, C., \& Castillo-Rogríguez, A. (2020). Attitude vs. Aptitude. Effect of psychological responses on soccer referees. International Journal of Sport Psychology, 51(1), 69-81. https://doi.org/10.7352/ IJSP.2019.50

O'Donoghue, P. (2013). Statistics for sport and exercise studies: An introduction. New York: Routledge. https:// doi.org/10.4324/9780203133507

Onetti-Onetti, W., Álvarez-Kurogi, L., \& Castillo-Rodríguez, A. (2019). Adherence to the Mediterranean diet pattern and self-concept in adolescents. Nutrición Hospitalaria, 36(3), 658-664. http://dx.doi. org/10.20960/nh.2214

Owen, A. L., Lago-Peñas, C., Gómez, M. Á., Mendes, B., \& Dellal, A. (2017). Analysis of a training mesocycle and positional quantification in elite European soccer players. International Journal of Sports Science and Coaching, 12(5), 665-676. https://doi. org/10.1177/1747954117727851

Payyappalli, V. M., \& Zhuang, J. (2019). A data-driven integer programming model for soccer clubs'decision making on player transfers. Environment Systems and Decisions, 39(4), 466-481. https://doi.org/10.1007/ s10669-019-09721-7

Petersen, C., Pyne, D., Portus, M., \& Dawson, B. (2009). Validity and reliability of GPS units to monitor cricket-specific movement patterns. International Journal of Sports Physiology and Performance, 4(3), 381393. https://doi.org/10.1123/ijspp.4.3.381

Randers, M. B., Nielsen, J. J., Krustrup, B. R., Sundstrup, E., Jakobsen, M. D., Nybo, L., \& Krustrup, P. (2010). Positive performance and health effects of a football training program over 12 weeks can be maintained over a 1-year period with reduced training frequency. Scandinavian Journal of Medicine \& Science in Sports, 20, 80-89. https://doi.org/10.1111/j.1600-0838.2010.01091.X

Rodrigo, G., Lusiardo, M., \& Pereira, G. (1990). Relationship between anxiety and performance in soccer players. International Journal of Sport Psychology, 21(2), 112-120.
Rosenberg, M. (1965). Society and the adolescent self image. Princeton NJ: Princeton University Press. https://doi.org/10.1515/9781400876136

Rosnet, E. (2000). Coping strategies and performance in fencing. International Journal of Psychology, 35(3-4), 232-232

Sandín, B., Chorot, P., Lostao, L., \& Valiente, R.M. (2012). Screening del cáncer de mama: Afectación psicológica. UNED.

Scherr, J., Wolfarth, B., Christle, J. W., Pressler, A., Wagenpfeil, S., \& Halle, M. (2013). Associations between Borg's rating of perceived exertion and physiological measures of exercise intensity. European Journal of Applied Physiology, 113(1), 147-155. https://doi. org/10.1007/s00421-012-2421-x

Tanaka, H., Monahan, K. D., \& Seals, D. R. (2001). Agepredicted maximal heart rate revisited. Journal of American College of Cardioly, 37(1), 153-156.

Vealey, R. S. (1986). Conceptualization of sport-confidence and competitive orientation: Preliminary investigation and instrument development. Journal of Sport Psychology, 8(3), 221-246. https://doi.org/10.1123/ jsp.8.3.221

Walker, A., Arent, M., McFadden, B., \& Arent, S. (2019). Physical Performance Testing in Soccer. Elite Soccer Players: Maximizing Performance and Safety, 137. https://doi.org/10.4324/9780429465536-8

Weinberg, R. S., \& Richardson, P. A. (1990). Psychology of Officiating. Champaign, IL.: Leisure Press. https:// doi.org/10.1037/0003-066X.45.12.1325

Woodman, T., \& Hardy, L. (2003). The relative impact of cognitive anxiety and self-confidence upon sport performance: A meta-analysis. Journal of Sports Sciences, 21(6), 443-457. https://doi.org/10.1080/0264041031000101809

Woolf, K., Bidwell, W. K., \& Carlson, A. G. (2009). Effect of caffeine as an ergogenic aid during anaerobic exercise performance in caffeine naive collegiate football players. Journal of Strength \& Conditioning Research, 23(5), 1363-1369. https://doi.org/10.1519/ JSC.0b013e3181b3393b
Este estudio ha sido financiado por el proyecto PPJIA2020.04, del programa Proyectos de Investigación Precompetitiva para Jóvenes Investigadores del Plan Propio 2020, de la Universidad de Granada. 